\title{
论 文＼cjkstart分子荧光探针专刊
}

\section{比率型亚硫酸氢根苂光探针的构建及其生物成像 研究}

\author{
张伟杰 ${ }^{1}$, 刘涛 ${ }^{1}$, 霍方俊 ${ }^{2}$, 宁鹏 $^{3}$, 温莹 ${ }^{1}$, 孟祥明 ${ }^{3}$, 阴彩霞 ${ }^{1 *}$ \\ 1. 生物化学和分子工程教育部重点实验室, 山西省能源材料转储重点实验室, 山西大学分子科学研究所, 太原 030006 \\ 2. 山西大学应用化学研究所, 太原 030006 \\ 3. 安徽大学化学化工学院, 合肥 230601 \\ *通讯作者, E-mail: yincx@sxu.edu.cn
}

收稿日期: 2017-03-21; 接受日期: 2017-05-02; 网络版发表日期: 2017-07-18

国家自然科学基金(编号: 21672131)和山西省人才支持计划(编号: 2014401)资助项目

\begin{abstract}
摘要本文基于菜并吡喃-吲哚盐共轭体系设计合成了能够检测亚硫酸氢根 $\left(\mathrm{HSO}_{3}{ }^{-}\right)$的新型近红外比率型 荧光探针. 该探针对 $\mathrm{HSO}_{3}{ }^{-}$识别显示出优异的选择性、较高的灵敏度和超快速的荧光响应. 加入 $\mathrm{HSO}_{3}{ }^{-}$后, 探 针展现出21.6倍 $\left(I_{520} / I_{653}\right)$ 的相对荧光强度的增强, 并伴随着溶液颜色由紫色转变为无色. 此外, 高分辨率质谱 和核磁氢谱滴定共同证实了该探针对 $\mathrm{HSO}_{3}{ }^{-}$的检测机制是 $\mathrm{HSO}_{3}{ }^{-}$与探针的不饱和碳碳双键发生了亲核加成 反应。最后, 探针被成功地应用于活细胞和斑马鱼幼体中 $\mathrm{HSO}_{3}^{-}$的原位示踪.
\end{abstract}

关键词近红外, 比率型, 亚硫酸氢根, 荧光探针, 生物成像

\section{1 引言}

作为主要的大气污染物之一, 二氧化硫 $\left(\mathrm{SO}_{2}\right)$ 极易 溶于水并转变成亚硫酸盐和亚硫酸氢盐 $\left(\mathrm{HSO}_{3}{ }^{-}\right)$, 对人 体健康和生态环境造成严重影响 ${ }^{[1 \sim 7]}$. 同时, 亚硫酸氢 钠广泛应用于食品添加剂, 由于其潜在的细胞毒性, 因此对其添加量的控制必须要有严格的要求. 美国 食品药品监督管理局已经明确规定, 食品标签上应注 明 $\mathrm{HSO}_{3}{ }^{-}$的含量, 且其含量不得超过 $10 \mu \mathrm{g} / \mathrm{mL}^{[8-11]}$. 因 此, 开发有效的方法用于监测 $\mathrm{HSO}_{3}{ }^{-}$具有极为重要的 意义. 苂光探针由于兼具高灵敏度、实时监测和操作 简便等优点, 在环境和生物系统中检测待分析物时具 有明显的优势 ${ }^{[12,13]}$. 已有很多能够专一性识别 $\mathrm{HSO}_{3}^{-}$ 的探针被设计并开发, 其中还包括一些可以靶向定位
的 ${ }^{[14-16]}$ 和可逆的 ${ }^{[17]}$ 探针. 然而, 这些探针中绝大多数 都是属于荧光增强型(turn-on). 而与这种单一发射波 长变化的探针相比, 比率型苂光探针由于具有双波长 发射特征、受环境因素影响小、响应范围宽、准确 度高且可以实现半定量分析等特性 ${ }^{[18-22]}$, 而受到广泛 关注. 目前识别 $\mathrm{HSO}_{3}{ }^{-}$的比率型苂光探针比较少. 在 本文中, 基于半花菁染料开发设计了具有较高选择性 和灵敏度, 能够用于专一性检测溶液、细胞及活体中 $\mathrm{HSO}_{3}{ }^{-}$的比率型苂光探针 1 (图 1).

\section{2 实验部分}

所有实验药品除非特别说明, 均购自阿拉丁试剂 公司(美国), 各种分析物包括 $\mathrm{Na}_{2} \mathrm{SO}_{3}$ 和 $\mathrm{NaHSO}_{3}$ 购自上

引用格式: 张伟杰, 刘涛, 霍方俊, 宁鹏, 温莹, 孟祥明, 阴彩霞. 比率型亚硫酸氢根苂光探针的构建及其生物成像研究. 中国科学: 化学, 2017, 47: 1022-1028 Zhang W, Liu T, Huo F, Ning P, Wen Y, Meng X, Yin C. A ratiometric fluorescent probe for sensing bisulphate and its application in living cells and zebrafish. Sci Sin Chim, 2017, 47: 1022-1028, doi: 10.1360/N032017-00057 


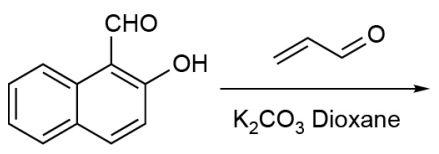<smiles>O=CC1=Cc2c(ccc3ccccc23)OC1</smiles>

2

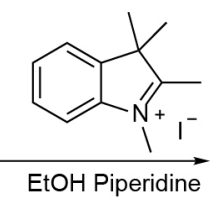

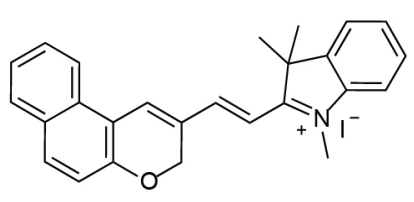

Probe 1

图 1 探针 1 的合成路线

海实验试剂有限公司 (中国). 所有检测实验均在室 温 $\left(25^{\circ} \mathrm{C}\right)$, 二甲基亚砜-磷酸盐缓冲溶液(DMSO-PBS) (10.0 mM, pH 7.4, 1:1, v/v) 中进行.

U-3900 型紫外 - 可见光分光光度计 (Hitachi 公 司, 日本), F-7000型苂光光谱仪(Hitachi公司), Bruker AVANCE-600 MHz超导核磁共振波谱仪(德国), AB SCIEX Triple TOF 5600plus 高分辨飞行时间质谱仪 (美国), PO-120石英比色典(上海华美实验仪器厂, 中 国), GL-9406型手提式紫外分析仪(郑州明天仪器有 限公司, 中国), 梅特勒-托利多 $\mathrm{pH}$ 计 (梅特勒, 瑞士), Olympus FV1200激光共聚焦扫描显微镜(日本).

\section{3 结果与讨论}

\section{1 探针 1 的合成与表征}

探针1的合成(图1). 取化合物 $2(0.105 \mathrm{~g}, 0.5 \mathrm{mmol})$ 和 1,2,3,3-四甲基- $3 H$-吲哚鎓碘化物 $(0.21 \mathrm{~g}, 0.7 \mathrm{mmol})$ 溶于 $10 \mathrm{~mL}$ 无水乙醇中, 加入催化剂哌啶 $30 \mu \mathrm{L} .78^{\circ} \mathrm{C}$ 搅拌回流 $12 \mathrm{~h}$, 然后冷却至室温, 过滤并用冰乙醇洗涤 得墨绿色固体, 真空干燥. 产量为 $0.202 \mathrm{~g}$, 产率为 $82 \%$. 核磁共振氢谱 $\left.{ }^{1} \mathrm{H} \mathrm{NMR}\right)(600 \mathrm{MHz}, \mathrm{DMSO}) \delta 8.64$ (s, $1 \mathrm{H}), 8.32$ (d, $J=16.1 \mathrm{~Hz}, 1 \mathrm{H}), 8.23$ (d, $J=8.5 \mathrm{~Hz}, 1 \mathrm{H}), 8.02$ $(\mathrm{d}, J=8.9 \mathrm{~Hz}, 1 \mathrm{H}), 7.95(\mathrm{~d}, J=8.1 \mathrm{~Hz}, 1 \mathrm{H}), 7.91 \sim 7.84(\mathrm{~m}$, 2H), $7.69(\mathrm{t}, J=7.6 \mathrm{~Hz}, 1 \mathrm{H}), 7.65 \sim 7.58(\mathrm{~m}, 2 \mathrm{H}), 7.51(\mathrm{t}$, $J=7.5 \mathrm{~Hz}, 1 \mathrm{H}), 7.25$ (d, $J=8.9 \mathrm{~Hz}, 1 \mathrm{H}), 6.97$ (d, $J=16.2 \mathrm{~Hz}$, $1 \mathrm{H}), 5.43(\mathrm{~s}, 2 \mathrm{H}), 4.09(\mathrm{~s}, 3 \mathrm{H}), 1.81(\mathrm{~s}, 6 \mathrm{H})$. 核磁共振 碳谱 $\left({ }^{13} \mathrm{C}\right.$ NMR) (151 MHz, DMSO) $\delta 180.10$ (s), 154.73 (s), 149.52 (s), 142.79 (s), 141.30 (s), 135.50 (s), 133.71 (s), 129.62 (s), 128.49 (s), 127.99 (s), 127.63 (s), 124.30 (s), 122.24 (s), 120.94 (s), 116.86 (s), 114.90 (s), 114.30 (s), 110.74 (s), 63.45 (s), 51.17 (s), 33.51 (s), 24.86 (s). ESI-MS: $\mathrm{m} / \mathrm{z}$ Calcd 366.1862, Found 366.1858.

化合物 2 的合成. 将丙烯醛 $(0.5 \mathrm{~mL}, 7.5 \mathrm{mmol})$ 和
2-羟基-1-䒬甲醛 $(0.516 \mathrm{~g}, 3.0 \mathrm{mmol})$ 溶解在含有碳酸 钾 $(1.035 \mathrm{~g}, 7.5 \mathrm{mmol})$ 的 1,4 -二氧六环溶液中. 将混合 物在 $105^{\circ} \mathrm{C}$ 加热反应 $72 \mathrm{~h}$. 反应完成后, 冷却至室温, 倒入冰水中搅拌 $30 \mathrm{~min}$. 混合物用乙酸乙酯萃取 3 次. 合并有机层并用饱和氯化钠洗涤两次, 然后用无水 硫酸钠干燥, 减压旋转蒸发除去溶剂, 所得粗产物经 硅胶柱色谱分离纯化, 洗脱剂为乙酸乙酯和石油醚 $(v / v, 1: 5)$ 混合液, 得到浅黄色固体 2 , 质量为 $0.126 \mathrm{~g}$, 产 率为 $20 \%$. ${ }^{1} \mathrm{H} \mathrm{NMR}(600 \mathrm{MHz}, \mathrm{DMSO}) \delta 9.72(\mathrm{~s}, 1 \mathrm{H})$, 8.46 (s, 1H), 8.24 (d, J=8.4 Hz, 1H), 8.00 (d, J=8.9 Hz, 1H), 7.92 (d, $J=8.1 \mathrm{~Hz}, 1 \mathrm{H}), 7.65$ (t, $J=7.6 \mathrm{~Hz}, 1 \mathrm{H}), 7.48$ (t, $J=7.5 \mathrm{~Hz}, 1 \mathrm{H}), 7.19$ (d, $J=8.9 \mathrm{~Hz}, 1 \mathrm{H}), 5.06$ (s, 2H). ${ }^{13} \mathrm{C}$ NMR (151 MHz, DMSO) $\delta 190.9$ (s), 155.4 (s), 138.4 (s), 134.5 (s), 130.8 (s), 129.9 (s), 129.5 (s), 129.2 (s), 128.6 (s), 125.1 (s), 122.1 (s), 117.9 (s), 114.4 (s), 63.0 (s) . ESI-MS: $m / z$ Calcd 211.0754, Found 211.0755.

\section{2 光谱性能研究}

\subsection{1 探针 1 对 $\mathrm{HSO}_{3}{ }^{-}$的紫外、苂光光谱滴定研究}

首先, 测试了探针 $\mathbf{1}$ 的紫外吸收和荧光发射光谱. 图2(a)显示当将 $\mathrm{HSO}_{3}{ }^{-}$溶液加入到含有探针 $\mathbf{1}(10 \mu \mathrm{M})$ 的DMSO:PBS缓冲液 $(1: 1, v / v, \mathrm{pH} 7.4)$ 中时, 对应紫外 吸收光谱的变化. 随着 $\mathrm{HSO}_{3}{ }^{-}$浓度 $(0 \sim 40 \mu \mathrm{M})$ 的增加, 探 针在 $520 \mathrm{~nm}$ 处的吸收峰逐渐减小, $350 \mathrm{~nm}$ 处的吸收峰 逐渐增强, 并且在 $360 \mathrm{~nm}$ 处形成了等吸收点. 这表明 $\mathrm{HSO}_{3}{ }^{-}$的加入, 使探针生成了新的化合物. 溶液颜色从 红色变为无色, 表明探针 1 可以实现 “裸眼”检测 $\mathrm{HSO}_{3}{ }^{-}$.

相应的苂光光谱变化如图2(b)所示, 探针 1本身在 $653 \mathrm{~nm}$ 处显示近红外苂光发射. 随着 $\mathrm{HSO}_{3}{ }^{-}(0 \sim 40 \mu \mathrm{M})$ 的加入, 探针 $\mathbf{1}$ 在 $653 \mathrm{~nm}$ 处的荧光强度逐渐降低, 伴随 有 $520 \mathrm{~nm}$ 处的荧光强度显著增强, 表明探针的 $\pi$ 共轭被 破坏. 此外, 如图2(c) 所示, $520 \mathrm{~nm}$ 处与 $653 \mathrm{~nm}$ 处相对 苂光强度的比值 $\left(I_{520} / I_{653}\right)$ 与 $\mathrm{HSO}_{3}{ }^{-}$浓度 $(0 \sim 40 \mu \mathrm{M})$ 呈现 

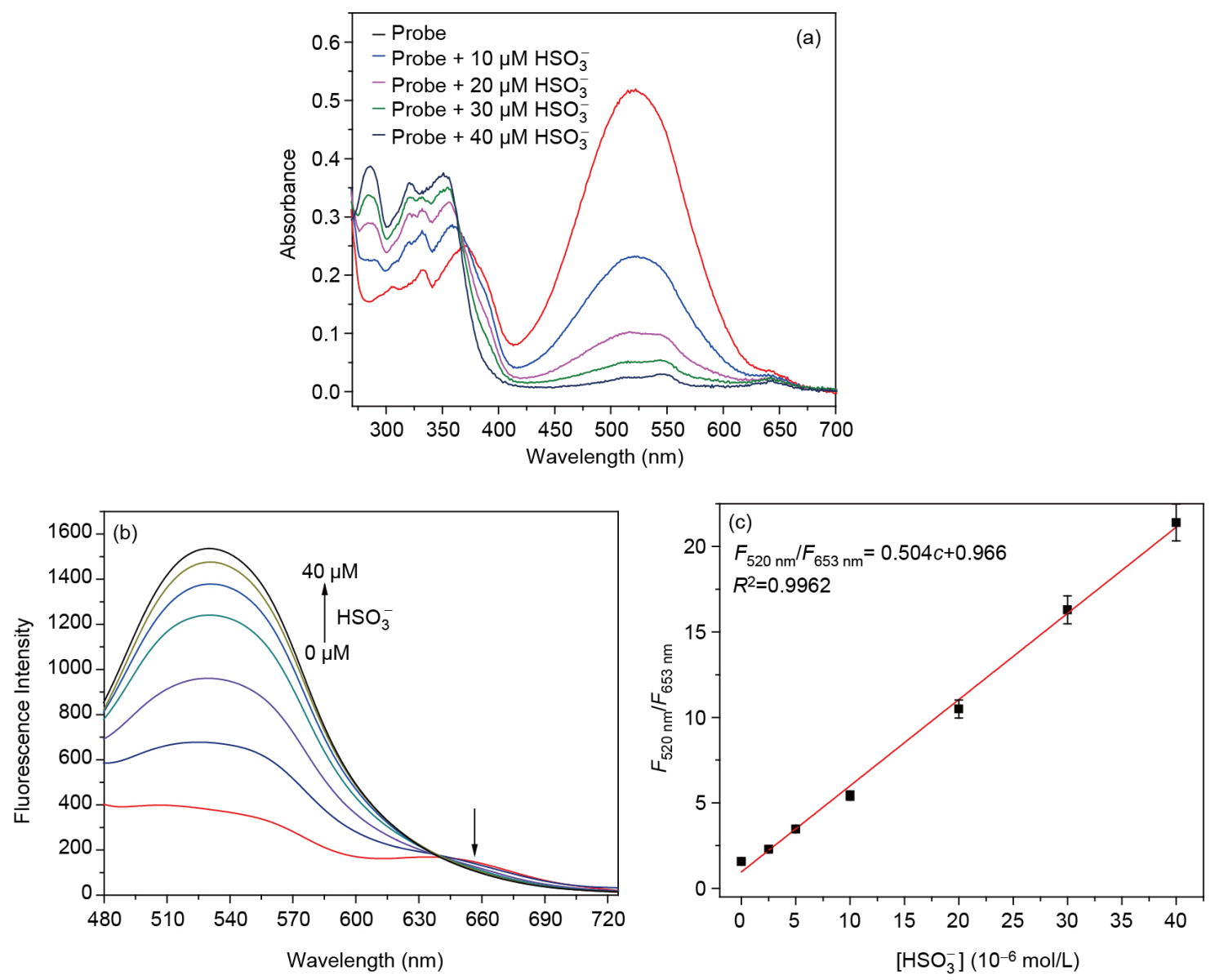

图 2 探针 $\mathbf{1}(10 \mu \mathrm{M})$ 随 $\mathrm{HSO}_{3}^{-}(0 \sim 40 \mu \mathrm{M})$ 浓度变化的紫外-可见吸收光谱图(a)和苂光发射光谱图(b); (c) 探针 1 与 $\mathrm{HSO}_{3}^{-}$作用后 相对荧光强度与浓度的线性关系, 激发波长为 $402 \mathrm{~nm}$, 狭缝: $5 \mathrm{~nm} / 10 \mathrm{~nm}$ (网络版彩图)

良好的线性相关 $\left(R^{2}=0.9962\right)$. 利用国际纯粹和应用 化学联合会(IUPAC) 给出的检出限计算公式 $(\mathrm{CDL}=$ $\left.3 S_{\mathrm{b}} / m\right)^{[23-26]}$ 可知, 探针 1 对 $\mathrm{HSO}_{3}^{-}$的检测限为 $76 \mathrm{nM}$. 这明显低于目前已经报道的类似结构的荧光探针 ${ }^{[27-29]}$.

\subsection{2 探针1对 $\mathrm{HSO}_{3}{ }^{-}$的选择性识别研究}

探针的重要特征是对于一种分析物具有特异性 识别功能. 为了检测探针 1 对 $\mathrm{HSO}_{3}^{-}$的特异性识别能 力, 考察了在DMSO:PBS (1:1, v/v, pH 7.4)缓冲液中加 入 $\mathrm{HSO}_{3}{ }^{-}(40 \mu \mathrm{M})$ 和其他分析物 $(400 \mu \mathrm{M})$ 后荧光强度比 值的变化(图3). 我们发现, 在 $402 \mathrm{~nm}$ 的激发波长下, 只 有 $\mathrm{SO}_{3}{ }^{2-}$ 和 $\mathrm{HSO}_{3}{ }^{-}$的加入引起了明显的相对荧光强度 $\left(I_{520} I_{653}\right)$ 变化, 而其他阴离子没有引起明显的荧光强度 比值变化. 该结果证明探针 1 对 $\mathrm{HSO}_{3}{ }^{-}$具有良好的选择 性识别能力.

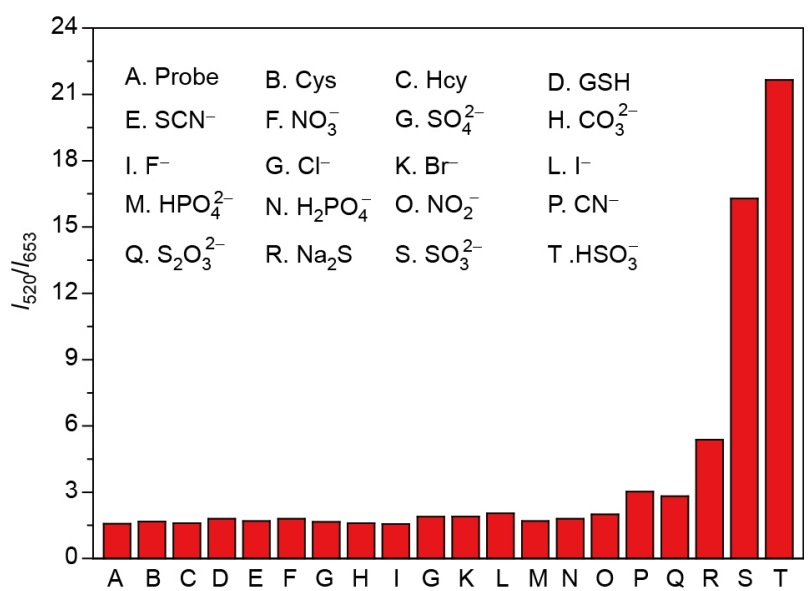

图 3 探针 1 与各类分析物作用后的相对苂光强度比值 $\left(I_{520} / I_{653}\right)$ 变化(网络版彩图)

\subsection{3 探针 1 识别 $\mathrm{HSO}_{3}{ }^{-}$的最适 $\mathrm{pH}$ 研究}

我们对探针1在不同 $\mathrm{pH}(2.0 \sim 9.0)$ 条件下的识别性 
能做了进一步研究(图4). 结果表明, 探针 $1(10 \mu \mathrm{M})$ 本 身的荧光强度比值 $\left(I_{520} / I_{653}\right)$ 在 $\mathrm{pH}$ 2.0 9.0 基本保持不 变. 在加入 $\mathrm{HSO}_{3}^{-}(40 \mu \mathrm{M})$ 后, 探针1在 $\mathrm{pH} 5 \sim 9$ 范围内的 荧光强度比值 $\left(I_{520} / I_{653}\right)$ 明显增加. 并且在生理环境 $(\mathrm{pH}$ 7.4)下具有良好的稳定性和识别效果. 因此, 预测探针 1 能够在生理条件下完成对 $\mathrm{HSO}_{3}^{-}$的识别检测.

\subsection{4 探针 1 对 $\mathrm{HSO}_{3}{ }^{-}$识别动力学研究}

此外, 考察了探针 1 对 $\mathrm{HSO}_{3}^{-}$的动力学响应过程, 如图5所示, 在 $400 \mu \mathrm{M} \mathrm{HSO}_{3}{ }^{-}$存在下, 探针 $\mathbf{1}$ 与 $\mathrm{HSO}_{3}{ }^{-}$的 反应在 $20 \mathrm{~s}$ 内基本完成, 并且苂光强度在 $1 \mathrm{~min}$ 内基本 保持稳定, 表明探针 1 能够快速地对 $\mathrm{HSO}_{3}{ }^{-}$进行检测.

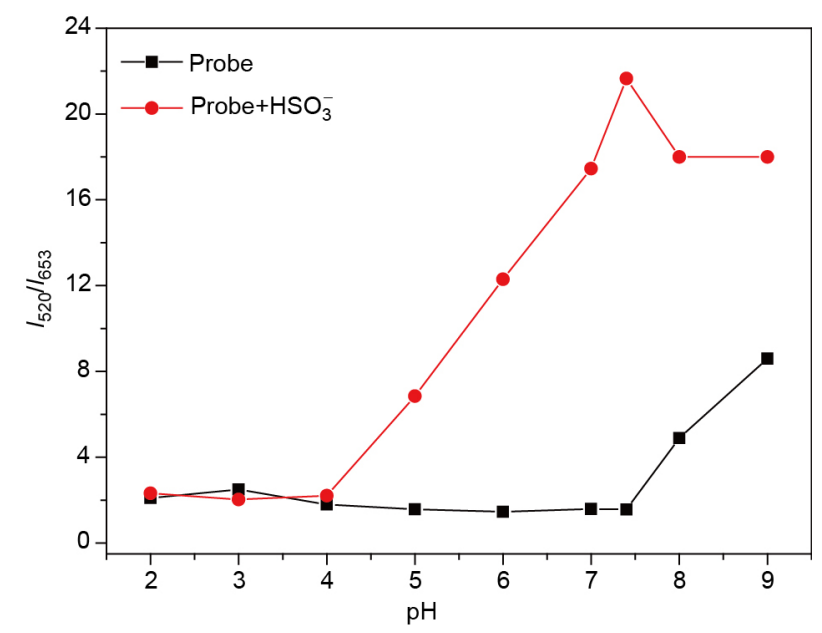

图 4 探针 1 与 $\mathrm{HSO}_{3}{ }^{-}$在不同 $\mathrm{pH}$ 条件下苂光强度比值 $\left(I_{520} / I_{653}\right)$ 的变化(网络版彩图)

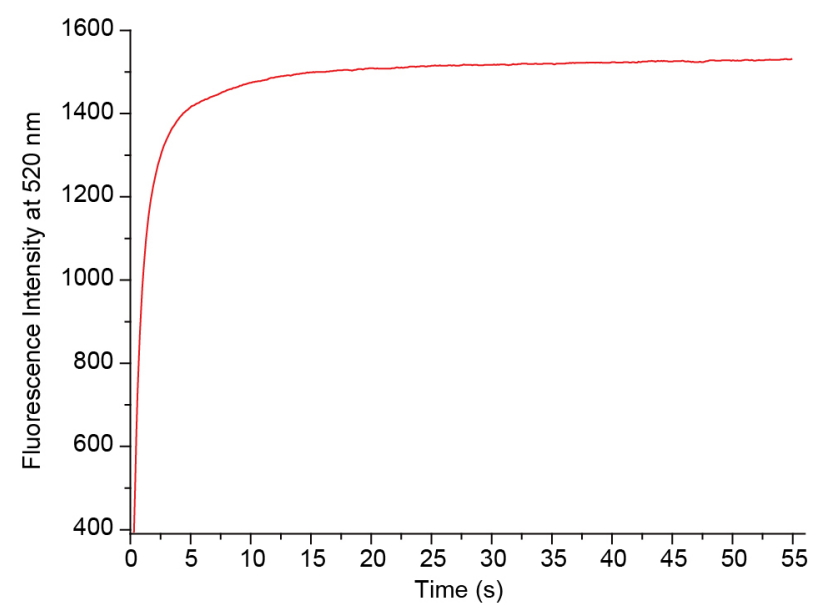

图 5 探针 1 与 $\mathrm{HSO}_{3}^{-}$作用的动力学研究(网络版彩图)

\section{3 探针 1 对 $\mathrm{HSO}_{3}{ }^{-}$识别机理的研究}

为了进一步证实探针 1 对 $\mathrm{HSO}_{3}{ }^{-}$的响应机理 (图6(a)), 选择核磁、质谱等分析手段进行研究. 当在 探针储备液 (DMSO- $\left.d_{6}\right)$ 中加入 $\mathrm{HSO}_{3}{ }^{-}\left(\mathrm{D}_{2} \mathrm{O}\right)$ 时, 发现 $\delta$ $8.62(\mathrm{a} 1) 、 \delta 5.42(\mathrm{~b} 1)$ 和 $\delta 4.09(\mathrm{e} 1)$ 处的 $\mathrm{H}$ 质子向高场 移动( $\mathrm{a} 2 、 \mathrm{~b} 2 、 \mathrm{e} 2$ 分别在 $\delta 7.08, \delta 3.09$ 和 $\delta 3.03 \mathrm{ppm}$ ). 同时探针 1 碳碳双键的 $\mathrm{H}$ 质子 $(\mathrm{c} 1,7.26,7.24$ 和 $\mathrm{d} 1,6.98$, $6.95 \mathrm{ppm}$ ) 也分别转移到 $5.37,5.35$ 和 $5.30,5.28 \mathrm{ppm}, \mathrm{c} 2$, $\mathrm{d} 2$ (图 6(b)). 此外, 从探针 1 与 $\mathrm{HSO}_{3}{ }^{-}$反应后的质谱结 果可以发现, 质谱中两个主要峰366.18和448.15分别 归于 $[\mathrm{M}]^{+}$和 $\left[\mathrm{M}+\mathrm{HSO}_{3}{ }^{-}\right]^{+}$(图6(c)). 由此表明, 探针 1 对 $\mathrm{HSO}_{3}{ }^{-}$的响应机制是基于图6(a)所示的亲核加成反应.

\section{4 细胞成像和斑马鱼成像研究}

为了检测探针 $\mathbf{1}$ 的细胞通透性和选择性识别细胞内 $\mathrm{HSO}_{3}{ }^{-}$的能力, 探针 1 被用于活细胞成像研究. 如图7所 示, 将探针 $\mathbf{1}(10 \mu \mathrm{M})$ 与人乳腺癌细胞 $(\mathrm{MCF}-7)$ 在 $37^{\circ} \mathrm{C}$ 下 孵育 $30 \mathrm{~min}$, 此时在红色通道中显示红色荧光, 而绿色 通道只有很微弱的荧光信号. 由此表明, 探针 $\mathbf{1}$ 具有良 好的细胞通透性. 此外, 在对照实验中, 将 $\mathrm{HSO}_{3}{ }^{-}$与探 针1预处理过的MCF-7细胞再温育 $30 \mathrm{~min}$ 后, MCF-7细 胞显示较强的绿色荧光, 同时红色通道的苂光基本消 失. 结果表明, 探针能够检测活细胞中外源性的 $\mathrm{HSO}_{3}^{-}$.

为了进一步研究探针 $\mathbf{1}$ 检测活体内 $\mathrm{HSO}_{3}{ }^{-}$的浓度 变化, 将探针 1 进行了活体成像实验. 选用斑马鱼作为 实验对象, 如图8所示, 当斑马鱼与探针 $1(10 \mu \mathrm{M})$ 捊育 $30 \mathrm{~min}$ 时, 发现红色通道中显示出的明亮红色荧光, 而 在绿色通道中基本上没有苂光. 相比之下, 当用探针 1 预处理过的斑马鱼再与 $\mathrm{HSO}_{3}{ }^{-}$一起睬育 $30 \mathrm{~min}$ 后, 之 前红色通道中的红色荧光显著的减少, 绿色通道表现 出强烈的绿色荧光. 结果表明, 探针 $\mathbf{1}$ 可以用于检测斑 马鱼幼体体内的 $\mathrm{HSO}_{3}^{-}$.

\section{4 结论}

综上所述, 设计并合成了一种新型比率型荧光探 针用于识别 $\mathrm{HSO}_{3}{ }^{-}$. 该探针具有较好的选择性、较高的 灵敏度以及较宽的 $\mathrm{pH}$ 响应范围. 此外, 活细胞成像和 斑马鱼幼体成像表明, 探针 1 具有较好的细胞穿透性, 并 且可以用于检测生物体内 $\mathrm{HSO}_{3}{ }^{-}$浓度的变化. 研究结果 为设计其他生物分子的检测和成像提供了有益的参考. 
(a)

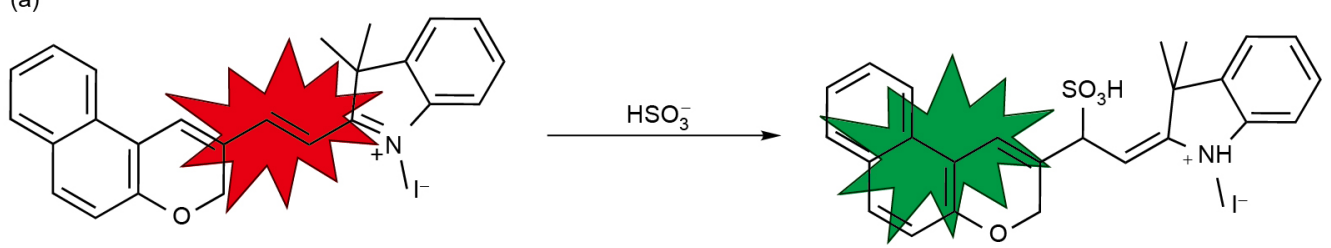

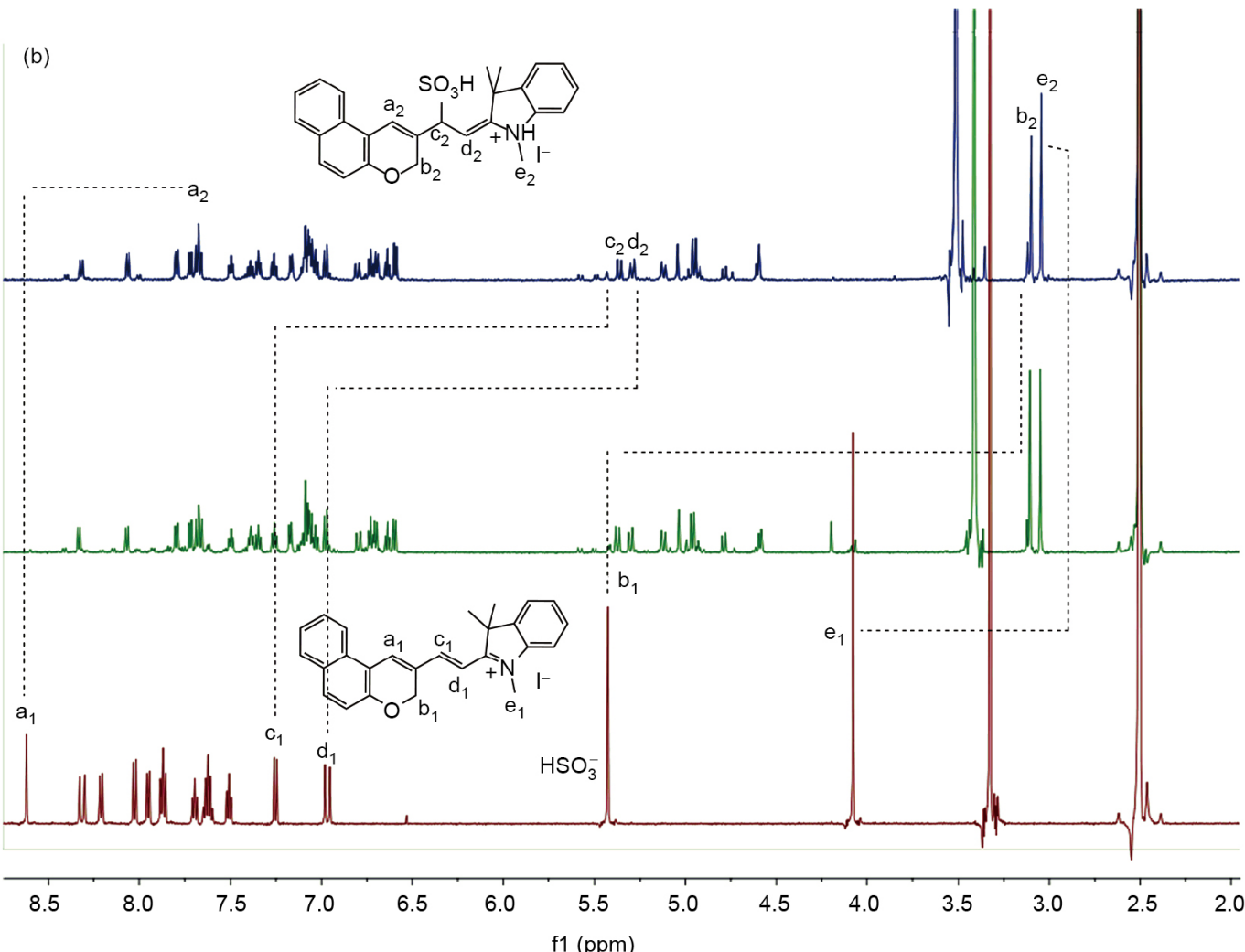

(c)

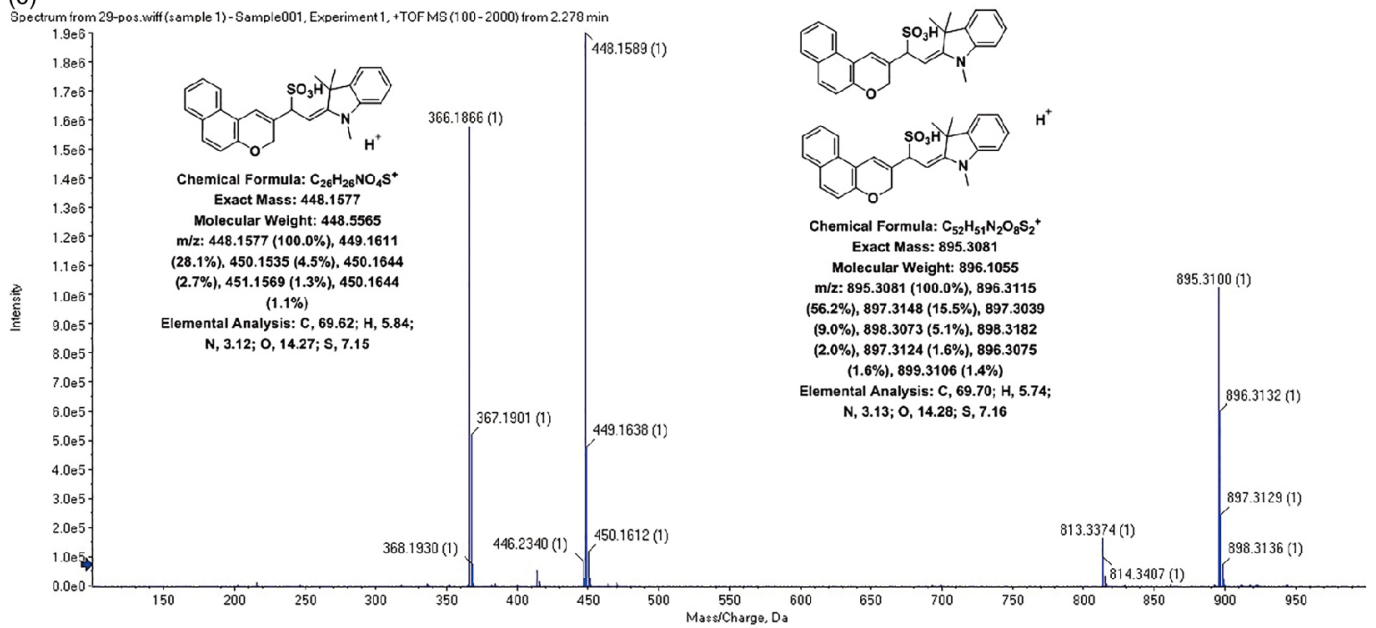

图 6 (a) 探针 1 对 $\mathrm{HSO}_{3}^{-}$的苂光响应机理示意图; (b) 探针 1 与 $\mathrm{HSO}_{3}^{-}$加成产物质谱图; (c) 探针 1 与 $\mathrm{HSO}_{3}^{-}$加成产物质谱图(网 络版彩图) 

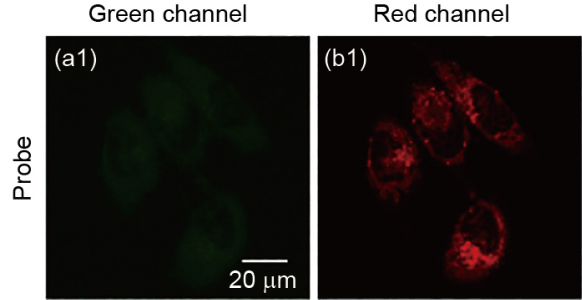

Bright field
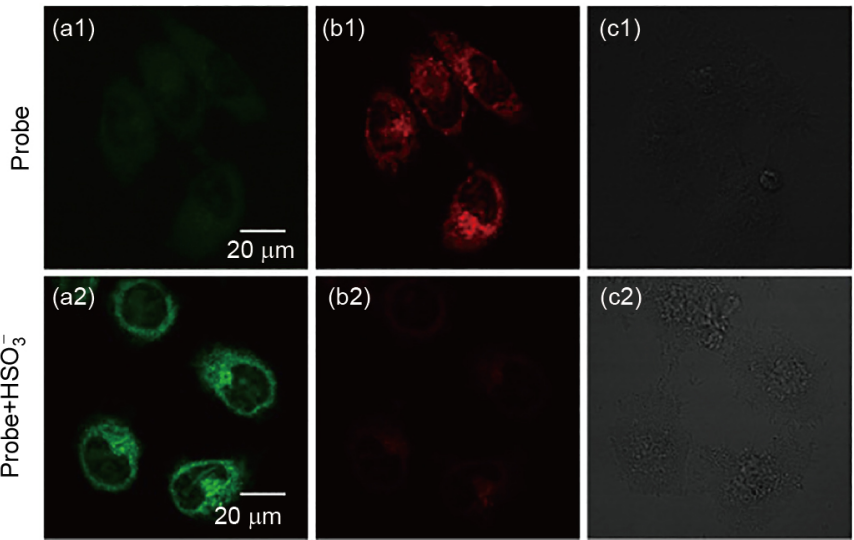

(b2)

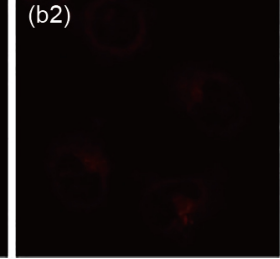

(c2)

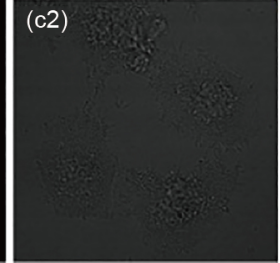

Merge

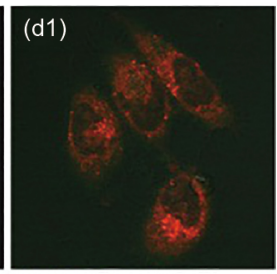

(d2)

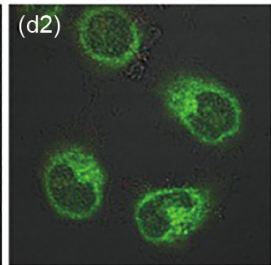

图 7 激光共聚焦细胞成像. (a1 d1) 探针 1 本身; (a2 $~ d 2)$ 探针 1 加入 $\mathrm{HSO}_{3}^{-}$后. (绿色通道: 激发波长 $405 \mathrm{~nm}$, 收集发射信号 $520 \pm 20 \mathrm{~nm}$. 红色通道: 激发波长 $488 \mathrm{~nm}$, 收集发射信号 $650 \pm 20 \mathrm{~nm}$ ) (网络版彩图)

Green channel

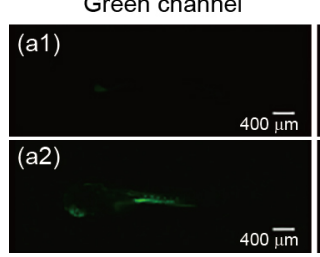

Red channel
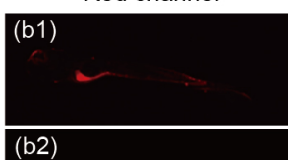

Bright field

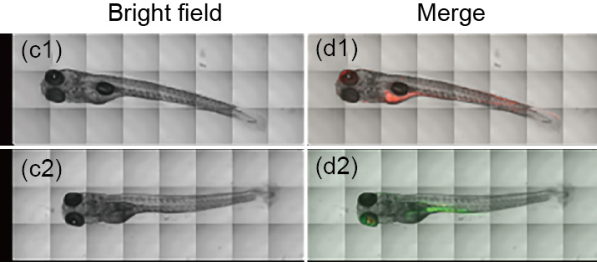

图 8 激光共聚焦斑马鱼成像. (a1 d1) 探针 1本身; (a2 d2) 探针 1 加入 $\mathrm{HSO}_{3}^{-}$后. (绿色通道: 激发波长 $405 \mathrm{~nm}$, 收集发射信号 $520 \pm 20 \mathrm{~nm}$. 红色通道: 激发波长 $488 \mathrm{~nm}$, 收集发射信号 $650 \pm 20 \mathrm{~nm}$ ) (网络版彩图)

\section{参考文献}

Meng Z, Qin G, Zhang B, Bai J. Mutagenesis, 2004, 19: 465-468

Shi X. J Inorg Biochem, 1994, 56: 155-165

Cardwell TJ, Christophersen MJ. Anal Chim Acta, 2000, 416: 105-110

Ekkad N, Huber CO. Anal Chim Acta, 1996, 332: 155-160

Fatibello-Filho O, da Cruz Vieira I. Anal Chim Acta, 1997, 354: 51-57

Leontiev AV, Rudkevich DM. J Am Chem Soc, 2005, 127: 14126-14127

Papkovsky D, Uskova MA, Ponomarev GV, Korpela T, Kulmala S, Guilbault GG. Anal Chim Acta, 1998, 374: 1-9

Fazio T, Warner CR. Addit Contam, 1990, 7: 433-454

9 Taylor SL, Higley NA, Bush RK. Adv Food Res, 1986, 30: 71-76

0 Yilmaz UT, Somer G. Anal Chim Acta, 2007, 603: 30-35

1 Sun Y, Zhao D, Fan S, Duan L, Li R. J Agric Food Chem, 2014, 62: 3405-3409

Chan J, Dodani SC, Chang CJ. Nat Chem, 2012, 4: 973-948

3 Liu C, Chen W, Shi W, Peng B, Zhao Y, Ma H, Xian M. J Am Chem Soc, 2014, 136: 7257-7260

4 Liu Y, Li K, Wu MY, Liu YH, Xie YM, Yu XQ. Chem Commun, 2015, 51: 10236-10239

5 Liu Y, Li K, Xie KX, Li LL, Yu KK, Wang X, Yu XQ. Chem Commun, 2016, 52: 3430-3433

6 Yang J, Li K, Hou JT, Li LL, Lu CY, Xie YM, Wang X, Yu XQ. ACS Sens, 2016, 1: 166-172

7 Zhang Y, Guan L, Yu H, Yan Y, Du L, Liu Y, Sun M, Huang D, Wang S. Anal Chem, 2016, 88: 4426-4431

8 Liu Z, Zhang C, Wang X, He W, Guo Z. Org Lett, 2012, 14: 4378-4381

Qu Y, Hua J, Tian H. Org Lett, 2010, 12: 3320-3323

20 Chen XY, Englert U, Bolm C. Chem Eur J, 2015, 21: 13221-13224 


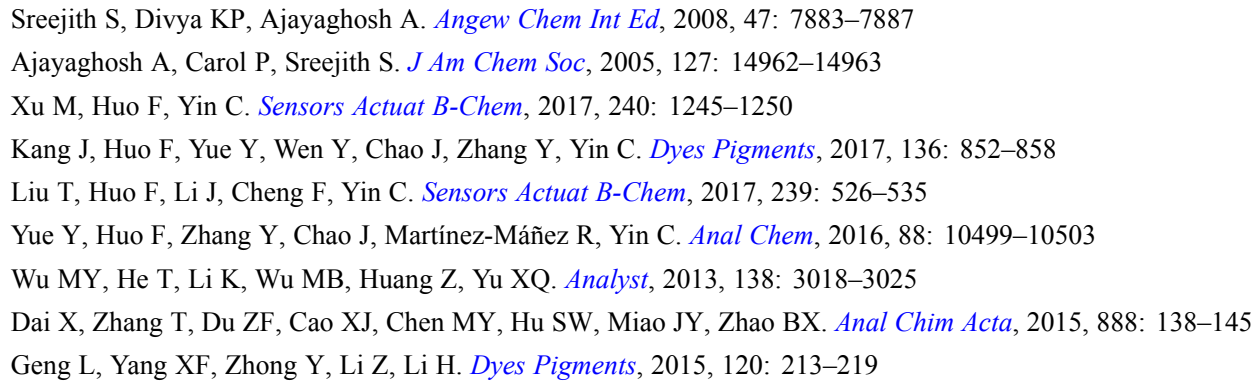

\title{
A ratiometric fluorescent probe for sensing bisulphate and its application in living cells and zebrafish
}

\author{
Weijie Zhang ${ }^{1}$, Tao Liu ${ }^{1}$, Fangjun $\mathrm{Huo}^{2}$, Peng Ning ${ }^{3}$, Ying Wen $^{1}$, Xiangming Meng ${ }^{3}$, Caixia Yin ${ }^{1 *}$ \\ ${ }^{1}$ Key Laboratory of Chemical Biology and Molecular Engineering of Ministry of Education, Key Laboratory of Materials for Energy Conversion and \\ Storage of Shanxi Province, Institute of Molecular Science, Shanxi University, Taiyuan 030006, China \\ ${ }^{2}$ Research Institute of Applied Chemistry, Shanxi University, Taiyuan 030006, China \\ ${ }^{3}$ Department of Chemistry, Anhui University, Hefei 230601, China \\ *Corresponding author (email: yincx@sxu.edu.cn)
}

\begin{abstract}
A novel near-infrared fluorescent probe for detection of bisulfite was designed and developed based on a conjugated naphthopyran-indolium system. The sensor showed excellent selectivity, high sensitivity and a rapid response toward bisulfite in aqueous solution. Upon the addition of $\mathrm{HSO}_{3}{ }^{-}$, the sensor displayed 21.6-fold $\left(I_{520} / I_{653}\right)$ fluorescence intensity enhancement, accompanied with an apparent color change from violet to colorless, suggesting that the sensor can be used to detect $\mathrm{HSO}_{3}{ }^{-}$with "naked-eye". The lowest detection limit for the above measurement was found to be $76 \mathrm{nM}$, and it was sensitive enough for the detection of endogenously produced bisulfite in biological system. Furthermore, the sensing mechanism was confirmed by high-resolution mass spectroscopy analysis and ${ }^{1} \mathrm{H}$ NMR spectrometry analysis, which was based on the strong nucleophilic addition reaction of bisulfite towards the sensor. Moreover, as a biocompatible molecule, the probe was successfully used for fluorescence imaging of bisulfite in MCF-7 cells and zebrafish, demonstrating that the probe is a novel ratiometric fluorescent probe to image $\mathrm{SO}_{2}$ derivatives in biosystem.
\end{abstract}

Keywords: near infrared, ratiometric, bisulfite/sulfite, fluorescent probes, bioimaging

doi: $10.1360 / \mathrm{N} 032017-00057$ 